\title{
Author Index to Volume 25
}

Aggett PJ, 167

Åkerblom, 323

Alaupovic P, 327

Albani M, 656

Alden BM, 46

Alessio M, 514

Amendt BA, 38

Amit $Y, 364$

Andreoli SP, 97

Andrew DS, 377

Andrew M, 405

Arab N, 107

Arab NL, 605

Arend R, 234

Ariagno RL, 258, 353

Armstead WM, 180

Armstrong D, 38

Auger D, 15

Aviram I, 276

Axelsson IA, 414

Aynsley-Green A, 553

Azzopardi D, 440, 445

Bailey PC, 457

Baldwin RB, 258

Balin A, 274

Bannon P, 519

Barstow TJ, 285

Bartlett K, 553

Baudin J, 445

Beasley DG, 180

Beisiegel U, 656

Bender BG, 577

Benderly A, 276

Bennet L, 573

Bensch K, 353

Bergstrom W, 262

Berry HK, 568

Bhutani VK, 143

Bidlingmaier F, 115

Bifano E, 262

Bignall S, 457

Birch-Machin MA, 553

Bohnsack JF, 634

Bortolussi R, 311

Bouillon R, 623

Bovee BW, 161

Brans YW, 377

Bratlid D, 205

Brauner R, 561

Bresson JL, 645

Bricker JT, 280

Bristow MR, 580

Brooker LA, 405

Brooks, JO III, 49

Brown KH, 503

Brunette MG, 15

Buescher ES, 266

Burbridge S, 311

Burhans M, 396

Burrows B, 316

Busija DW, 180

Cady EB, 440, 445

Cairo MS, 156

Carey KD, 377

Carpenter G, 535

Carter RL, 389

Cashore WJ, 452

Cha C-JM, 466
Chan G, 364

Chen B, 27

Chernick MS, 49

Chin T, 156

Chu SW, 225

Clark SJ, 347

Clausen M, 656

Clyman RI, 136

Cook C, 573

Cooper DM, 285

Corvol MT, 76

Covert RF, 389

Cowan BE, 27

Cpanao G, 514

Crissinger KD, 612

Daehne I, 353

Dahlström S, 323

Danks DM, 548

Darley-Usmar VM, 553

Dascalu V, 473

Davis L, 353

Degenhart HJ, 623

Delphy DT, 445

Delpy DT, 440

de Muinck Keizer-Schrama SMPF, 623

Devereaux MW, 55

DiGiacomo JE, 429

Dobbins SS, 617

Dobrozsi DJ, 339

Doerr $\mathrm{HG}, 115$

Dolan LM, 339

Donnell GN, 151

Dreyer WJ, 280

Drummond WH, 389

Dumontier MF, 76

Dunn JA, 360

Dutton EB, 377

Dynia DW, 239

Dzau VJ, 332

Eisenfeld L, 519

Elias W, 305

Ernst J, 496

Etzioni A, 276

Eylers JP, 617

Facchinetti F, 652

Faull RLM, 573

Fedunec S, 364

Feingold M, 336

Fernandes J, 327

Fewell JE, 473

Fike CD, 383

Filyk SC, 473

Fink RJ, 49

Fisher DE, 435

Fisher DJ, 280, 585

Fisher SE, 19

Fjeld CR, 503

Floros J, 239

Frank L, 399

Frates $\mathrm{RC} \mathrm{Jr}, 49$

Freeman BA, 525

Frenck RW, 266

Friedman AL, 509

Fukuda Y, 591

Gallaher KJ, 530
Garza C, 63, 184

Genazzani AR, 652

Gentile F, 514

Ghishan FK, 107, 189, 605

Gimotty PA, 389

Ginsburg V, 49

Glotzbach SF, 258

Gluckman PD, 573

Goebel HH, 89

Goldblum RM, 184

Goldman AS, 184, 629

Gore JC, 27

Gorter S, 573

Gourley GR, 234

Gourley MF, 234

Granger DN, 612

Gray ME, 535

Greca N, 519

Griese M, 209

Grisham MB, 612

Grondin G, 482

Grose WFA, 623

Gross I, 239

Grossman G, 130

Gruber HE, 202

Grumbach MM, 347

Guandalini S, 514

Guarino A, 514

Gunn AJ, 573

Hachey DL, 63

Hackeng WHL, 623

Hall C, 205

Hamilton PA, 440, 445

Hansen TH, 383

Hansen TWR, 205

Hardjowijono R, 44

Harzer K. 89

Hasu M, 274

Hauffa BP, 347

Hay S, 167

Hay WW Jr, 429

Heldt GP, 305

Hermos RJ, 336

Herrera E, 102

Herson VC, 519

Heymann MA, 136

Hick PJJ, 94

Hill HR, 634

Hirata Y, 591

Hoath SB, 542

Hochberg Z, 276

Hoeks APG, 94

Hoogenboezem T, 623

Hope PL, 440, 445

Huang M, 124

Hübner C, 656

Hughes TK Jr, 629

Hulman S, 124

Hulman SE, 219

Hung $\mathrm{HH}, 76$

Hyman PE, 600

Ichiki T, 194

Ikegami M, 32, 83, 130, 423

Ingelfinger JR, 332

Innis SM, 399

Inoue $F, 38$

Issekkutz T, 311

Jansons RA, 373
Jing J, 600

Jobe A, 130

Jobe AH, 32, 83, 423

Johnson MD, 525

Johnston BM, 573

Joyce JL, 409

Kalhan SC, 490

Kaplan SL, 347

Karl PI, 19

Kaufman FR, 151

Kavey R-E. 262

Keisari Y, 276

Kelch RP, 1

Khoury JC, 339

Kikuchi K, 107, 605

Kikuchi T, 107, 605

King KC, 490

Kinoshita Y, 6

Kliegman R, 124

Kliegman RM, 219

Kobavashi M, 194

Kobayashi Y, 6, 591

Koers JH, 44

Kohlschütter A. 656

Kojima T, 591

Kondo CS, 473

Koren G, 274

Krause PJ, 519

Kreutzer DL, 519

Kudlacz EM, 617

Kuipers JRG, 44

Kustermann-Kuhn B, 89

Lafond J, 15

Laitinen R, 323

Landau LI, 316

Langenbeck U, 11

Lappi SE, 617

Lawrence S, 173, 209

LeBel D, 478, 482

Leblond FA, 478, 482

Lee C, 452

Leffler CW, 180

Lemons J, 496

Levitsky LL, 435

Linden MG, 577

Linderkamp O, 595

Lindner SG, 656

Lissauer TJ, 457

Liu Y-M, 496

Lloyd DJ, 167

Lobo RA, 151

Lorch V, 360

Maddaiah VT, 119

Mak C, 580

Malone TA, 649

Mankin HJ, 76

Martin $G, 457$

Martin MG, 600

Masuda K, 6

Matherne GP, 291, 461

Matsuzaki S, 591

Mauray F, 136

Mayock DE, 27

McIlheran SM, 266

McWeeny OJ, 291

Merritt TA, 305

Meshulam T, 276 
Michael L, 280

Migliavacca M, 514

Mihalik S, 548

Miller PS, 119

Milligan DWA, 553

Millington DS, 38

Mirro R, 180

Mitchell L, 405

Mitchell ML, 336

Momma K, 69

Morgan WJ, 316

Morin FC III, 245, 251

Morisset J, 478, 482

Morris AH, 409

Moser HW, 548

Moses AC, 336

Moses L, 353

Mosier HD, 373

Mufson RA, 649

Mughal MZ, 420

Mushtaha AA, 629

Mydlar T, 573

Nakamura KT, 291, 461

Narcy P, 645

Navarro HA, 617

Neal P, 496

$\mathrm{Ng} W G, 151$

Nickerson PA, 251

Nielsen HC, 173

Nishikimi M, 194

Nonaka I, 194

Northway WH Jr, 353

Novello AC, 1

Novotny EJ Jr, 27

Odden J-P, 205

Ofosu FA, 405

Ohnishi T, 194

Ohtsu N, 353

Oh W, 452, 466

Oliven A, 490

Ozawa T, 194

Palta M, 234

Paridon SM, 280

Paton JB, 435

Peeters LL, 641

Penn RB, 143

Pepinsky RB, 535

Pergolizzi J, 262

Pesonen E, 305

Petraglia F, 652

Petriceks R, 353

Petroff OAC, 27

Pettenazzo A, 130, 423

Pickens WL, 542

Pityer R, 509
Polberger SKT, 414

Pollak S, 276

Porter C-BJ, 280

Possmayer F, 525

Poulos A, 548

Poznansky MJ, 364

Pratt RE, 332

Preston T, 167

Putet G, 645

Räihä NCR, 414

Rajaraman S, 629

Rampling MW, 457

Rannels DE, 530

Rannels SR, 530

Rappaport R, 76, 561

Reardon M, 280

Reeds PJ, 167

Reinhardt D, 209

Reiter EO, 409

Reneman RS, 94

Rey J, 645

Reynolds EOR, 440, 445

Rhead WJ, 38, 548

Rice WR, 396

Rich EA, 342

Rickard K, 496

Ricour C, 645

Rider ED, 83

Riedel BD, 189

Rimoin DL, 202

Rivers RPA, 457

Roberts DD, 49

Robertson B, 130

Robillard JE, 291, 461

Robinson A, 577

Rodens KP, 347

Rogers SR, 161

Roman C, 136, 228

Ross R, 420

Roth TP, 332

Rubino A, 514

Rudloff HE, 629

Rudolph AM, 228, 300

Rudolph CD, 228, 300

Ruffini L, 83, 423

Rusch NJ, 461

Sachs C, 645

Sahn DJ, 305

Saio MF, 634

Saunders SL, 161

Saxena PR, 641

Schanler RJ, 184

Schellekens $\mathrm{H}, 311$

Schiff D, 364

Schmalstieg FC, 629

Schmidt B, 405
Schoeller DA, 503

Schwartz CA, 377

Schwartz SA, 295

Seakins JWT, 11

Segal S, 161

Seidner S, 32, 83, 130

Seidner SR, 423

Sells SF, 542

Seo JK, 225

Shaddy RE, 580

Shaffer TH, 143

Shaul PW, 466

Shepherd IM, 553

Sherratt A, 553

Sherrill DL, 316

Shikes RH, 55

Sideropoulou O, 209

Silber GH, 63

Sinha SN, 360

Sippell WG, 115

Slade HB, 295

Slagle T, 262

Slotkin TA, 617

Smith DL, 496

Smith EO, 280

Smith FG, 291

Snape WJ Jr, 600

Sokol RJ, 55

Sosenko IRS, 399

Spirer Z, 276

Springer C, 285

Stack T, 167

Stahlman M, 535

Standaert TA, 27

Stanley H, 553

Stenzel W, 209

Stewart AL, 445

Stiris T, 205

Stolte G, 89

Stonestreet B, 452

Storchi AR, 652

Styne DL, 347

Sugiyama N, 194

Sundell H, 535

Suzuki H, 194

Sweeney TE, 353

Takao A, 69

Tanaka M, 194

Tansey PA, 258

Tanswell AK, 525

Tarallo L, 514

Taussig LM, 316

Tkaczevski $\mathrm{H}, 19$

Tomomasa T, 600

Toorop GP, 44

Townsend SF, 300

Toy C, 156

Traber MG, 55
Tran NN, 143

Trindade C, 124

Trippel SB, 76

Tsang RC, 420

Turnbull DM, 553

Tzehoval E, 276

VandeVen C, 156

van Kreel BK, 641

van Merode T, 94

van Straalen MJ, 44

Varille VA, 291

Verkeste CM, 641

Versmold HT, 115

Visser HKA, 623

Vorhees CV, 568

Vuori E, 323

Wada Y, 194

Walker WA, 225

Wallenburg HCS, 641

Warshaw J, 173

Warshaw JB, 209

Watkins PA, 548

Watmough NJ, 553

Weatherstone KB, 342

Weaver C, 496

Weisman S, 519

Weisman Y, 276

Welch RJ, 553

Wendel U, 11, 147

Whittingstall P, 457

Widness JA, 649

Wiehler U, 656

Wildevuur RH, 44

Wild LM, 251

Wilson CM, 239

Wispé JR, 396

Wit JM, 327

Wolfson MR, 143

Wong L, 525

Wong M, 27

Wong WW, 63

Wood PA, 38

Woodrum DE, 27

Wyatt JS, 440, 445

Xu Y-K, 151

Yamada T, 32, 83

Yang KD, 634

Young CA, 339

Young RSK, 27

Zakut V, 276

Zijlstra WG, 44

Zilow EP, 595

Zipursky A, 274

Zorzano A, 102

Zuckerman K, 27 